UCRL-1D-126456

\title{
Metrology of 13-nm Optics for Extreme Ultraviolet Lithography
}

\author{
J. F. Beckwith \\ S. R. Patterson \\ D. C. Thompson \\ Lawrence Livermore National Laboratory \\ V. Badami \\ S. Smith \\ University of North Carolina at Charlotte
}

February 3, 1997

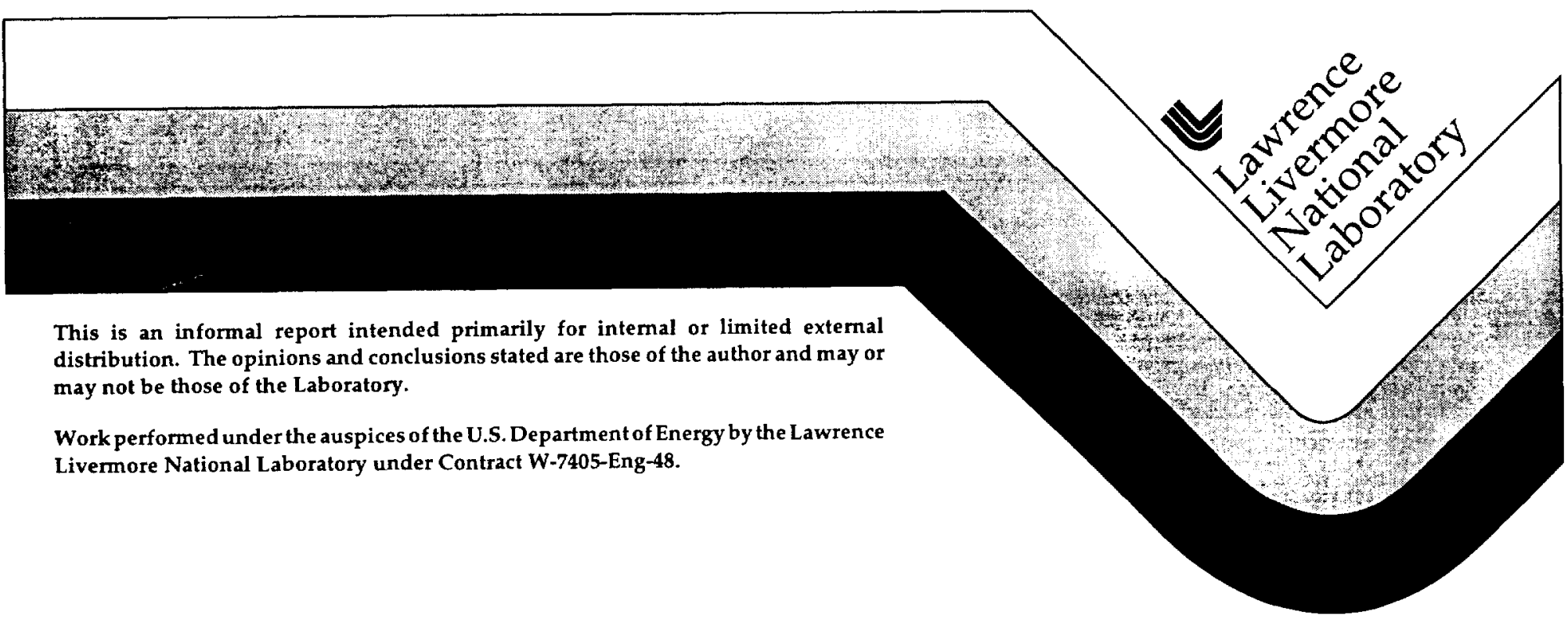




\section{DISCLAIMER}

This document was prepared as an account of work sponsored by an agency of the United States Government. Neither the United States Government nor the University of California nor any of their employees, makes any warranty, express or implied, or assumes any legal liability or responsibility for the accuracy, completeness, or usefulness of any information, apparatus, product, or process disclosed, or represents that its use would not infringe privately owned rights. Reference herein to any specific commercial product, process, or service by trade name, trademark, manufacturer, or otherwise, does not necessarily constitute or imply its endorsement, recommendation, or favoring by the United States Government or the University of California. The views and opinions of authors expressed herein do not necessarily state or reflect those of the United States Government or the University of California, and shall not be used for advertising or product endorsement purposes.

This report has been reproduced directly from the best available copy.

Available to DOE and DOE contractors from the Office of Scientific and Technical Information

P.O. Box 62, Oak Ridge, TN 37831

Prices available from (615) 576-8401, FTS 626-8401

Available to the public from the

National Technical Information Service

U.S. Department of Commerce

5285 Port Royal Rd.,

Springfield, VA 22161 


\title{
Metrology of 13-nm Optics for Extreme Uitraviolet
}

\section{Lithography}

\author{
J. Beckwith, S. Patterson, D. Thompson \\ Lawtence Livermore National Laboratory \\ V. Badami, S. Smith \\ University of North Carolina at Charlotte
}

\section{Introduction}

This report documents activities carriad in support of the design and construction of an ultra-high precision measuring machine intended for the support of Extreme Ultraviolet Lithography development. At the outset, this project was aimed at the overall fabrication of such a measuring machine. Shortly after initiation, however, the scope of activities was reduced and effort was concentrated on the key technical advances necessary to support such machine development: high-accuracy surface sensing and highly linear distance interferometry.

\section{Background}

Current semiconductor fabrication technology relies on contact photolithography to produce circuit features such as interconnecting lines and active solid-state devices. Device performance is improved and production oconomies are realized as the circuit feature size is reduced. Present photolithography techniques achieve feature size of the otder of $0.5 \mu \mathrm{m}$ ( $500 \mathrm{~nm}$ ) using diffraction-limited optical systems and illumination sources with $250 \mathrm{~nm}$ wavelength light. To achieve finer features will require the use of shorter wavelength sources. The next quantum step in semiconductor production technology is Extreme Ultraviolet Projection Lithography (EUVPL). EUVPL utilizes a source wavelength of order $13 \mathrm{~nm}$ to produce feature sizes substantially less than $0.1 \mu \mathrm{m}(100 \mathrm{~nm})$.

However, formidable technical challenges face the development of EUVPL. Proposod EUVPL systems use soveral near normal incidence multilayer-coated mildly aspheric mirrors. To meet the Rayleigh criteria for a diffraction-limited system implies that each mirror surface must achieve an accuracy of less than $0.3 \mathrm{~nm}$ rms, or about $1 \mathrm{~nm}$ peak-to-valley. This exceeds current optical metrology practice by more than an order of magnitude. While several potential EUVPL optical fabrication methods bave been proposed, these methods rely on differential measurements to achieve final accuracy. These methods can only be implemented when figure metrology with an accuracy exceeding $1 \mathrm{~nm}$ can be supplied. 
The basic requirements for accuracy and surface slope variation of the optics within an EUVPL system can be derived by considering the dimensional accuracy required to construct a diffraction-limited system at this wavelength. One commonly takes the definition of a diffraction-limited system to be one in which the Strehl ratio is greater than some assumed value. Since the Strehl ratio is, to a very good approximation in a nearly diffraction-limited system, a function of the mns wavefront error only, this provides a criterion for assigning an ms error to the wavefront. If the limit for the Strebl ratio is taken to be $80 \%$, then the allowable wavefiont error is approximately $\lambda / 10$ [']. If the optical system producing this wavefront consists of a single reflective clcment, then the specification for form error will be $2 / 20 \mathrm{rms}$. This sets the characteristic surface tolerance at $0.65 \mathrm{~nm}$ rms.

If the optical system consists of several elements which contribute aberrations to the wavefront in an uncorrelated way, then the allowable tolerance for any individual element will decrease as the square root of the number of elements. There is a practical limit, however, to the number of reflective elements which may used in the design of an Extreme Ultraviolet Projection Lithography (EUVPL) optical systcm. This limit is set by the upper limit to the achievable reflectivity of the multilayer surfaces. Currently available coatings have demonstrated reflectivity of approximately $60 \%$ ['] with somewhat lower values achieved on curved surfaces [']. If a $60 \%$ value is used for the optical system, then a six mirror optical design will result in a transmission of only $5 \%$, and a optical design providing less than an order of magnitude reduction in transmitted power will require that only four elements be used. With this in mind, the desirable rms figure accuracy of $0.33 \mathrm{~nm} \mathrm{~ms}[0.65 \mathrm{~nm} / \sqrt{4}]$ can be assigned to any single optical surface. This value has been assigned as the EUV Optics Measuring Machine accuracy goal.

Based on the range of curtent designs, we have assumed the maximum oplic to have a nearly circular cross-section with a maximum dimension of $250 \mathrm{~mm}$. Current designs appear to be limited 10 NA of 0.1 ( $f / 5)$ or less. We have, accordingly, taken the maximum sag to be that of a spherical optic of diameter $250 \mathrm{~mm}$ with radius of curvature of $1,250 \mathrm{~mm}$. Such an optic has a depth of $6.3 \mathrm{~mm}$. With this maximum sized optic to measure, the initial design measurement range has been set (somewhat arbitrarily) at $7.5 \mathrm{~mm}$ to permit some set up and auxiliary test range.

\section{Overall System Description}

The proposed EUVOMM combines high accuracy interferometry with atomic force microscopy for determination of absolute surface position. Interferometry is used to provide the high dynamic range required in the instrument $(1 \times 10-11 \mathrm{~m}$ in $7.5 \mathrm{~mm}$ of travel, or 1 part in $7.5 \times 108)$ with all time, frequency and distance measurements derived from a natural standard (atomic resonance). Atomic force microscopy (AFM) techniques permit the determination of the surface position with very low forces and probc-workpiece interactions that have been carefully studied by a number of researchers. The EUVOMM exploits a very high degree of mechanical repeatability to allow calibration of the measurement systems using inherently self-checking techniques.

A simplified conceptual design of the EUVOMM is shown in Figure 1. The machine consists of two principal subsystems: a vertical measurcment system attached to the base, and a scanning 
table which moves horizontally on the base. Each subsystem is provided with an appropriate drive, position feedback system and bearing. The vertical measurement systern is provided with both a full-travel low-bandwidth actuator and a short-travel high-bandwidth actuator capable of maintaining operation of the AFM probe in the desired region of its response. The short-travel actualor is also provided with the capability of making small changes in the angular orientation of the AFM assembly.

The two subsystems are connected by a metrology system, which includes a stable structure which provides for measurement of the relative positions between the two subsystems. Figure 2 schematically illustrates the use of a metrology frame to provide relative motion information in the $z$-direclion. The metrology frame provides a stable link between the $z$-interferometer assembly and transducers which measure the vertical distance between the metrology frame and the surface of the scan table. If the measured translation of the z-interferometer is $\mathrm{z}_{\text {inor }}$ and the two metrology frame transduccr values are $z_{m 1}$ and $z_{m 2}$, then the actual measurement of the workpiece in the absence of geometry errors in the machine is just $z_{i x}-0.5 \times\left(z_{m 1}+z_{m 2}\right)+c_{0}$, where $c_{0}$ is a constant which reflects the offset in the passive portions of the "metrology loop" connecting the AFM probe with the workpiece base. In fact, the actual calculation may be more complex, since the axis of the 2-interferometer may not be exactly centered between the metrology frame sensors. It should also be noted that the reference surface of the scan table (and hence $c_{0}$ ) will change as the scan table translates in the $\mathrm{x}$-direction.

The horizontal motion of the scan table is coupled to the vertical measurement subsystem using a laser interferometer which views both a reflector on the end of the table and a pair of reference reflectors attached to the moving vertical stage. This system works in a way analogous to the vertical metrology system. Bocause the position of the AFM tip will move vertically with respect to the plane containing the primary horizontal measurement and reference interferometer beams, a second interferometer is used to produce a measure of the tilt motion of the scan table so that the horizontal position of the AFM tip can be accurately measured or controlled.

Displacement measurement is provided by a set of heterodyne interferometers. These interferometers will provide at lease four passes of the optical beam in the measurement leg (configurations with more than four passes will also be investigated during the interferometer development phase). If readily available $633 \mathrm{~nm}$ wavelength laser sources are used, this provides a single cycle optical resolution of $160 \mathrm{~nm}$. The desired 0.01 um error contribution requires a phase measurement accuracy of 0.0004 radian (0.023 degrees). The interferometry system will achieve most of this phase resolution by direct phase measurement of each belerodyne signal cycle, with some resolution enhancement below the quantization level obtained by averaging the fluctuating signal. The use of the latter technique for resolution enhancement has been shown to provide at least a factor of 32 resolution enhancement and is the subject of a L,LNL-held patent.['] It should be straightforward to extend averaging over uniform fluctuation of reference to provide two orders of magnitude in resolution enhancement. Direct digital phase measurement has been previously implemented on the LODTM with a resolution of 0.025 radian.

Measurements with the present probe system bave shown that contact forces can be reliably maintained below $10^{-9}$ Newton. The measured noise level of the probe was $0.19 \mathrm{~nm} / \mathrm{VHz}$ This value was measured in an open laboralory with no acoustical shielding. Reducing this level by an order of magnitude is feasible and will require the introduction of low noise electronics and 
modifications to the positioning controller. In a modestly well contralled laboratory (0.1 C temperature control) and in the absence of specific thermal insulation, the probe repeatability has been measured by retracing a profile on a ZerodurTM specimen. This measure can be used to provide an estimate of the drift present in the probe. Using this measure, the probe has demonstrated drifl less than $2 \mathrm{~nm}$ (probably masked by surface roughness effects) over a period of 10 seconds, and $7 \mathrm{~nm}$ over a period of 5 minutes. A significant portion of the drift could be due to the reference capacitor, which had a higher thermal coefficient than the probe assembly itself. Reduction of drift to an acceptable level should be a straightforward, if exacting, task given the improved temperature control of the proposed instrument.

A larger challenge in the interferometer is eliminating systematic enors. The primary source of systematic error is mixing of unwanted polarization due to imperfection in the polarizing elements of the interferometer and quarter wave plates. Some systematic errors also arise from multiple reflections in the optics. Our approach to both errors is to minimize the intrinsic effect using high quality components and careful alignment, then correct the stable errors that remain. As a general design rule, we have set the improvement expected from correction to be between one and two orders of magnitude. Limiting the correction to two orders of magnitude is not done for physics reasons, but only to introduce some conscrvatism into the initial design.

Rosenbluth and Bobroff $\left[{ }^{5}\right]\left[{ }^{6}\right]$ have reviewed the major sources of non-linearity in beterodyne Michelson interferometers. Although non-linearities of 5-10 nm are common in commercial interferometers, their calculations show that values below $1 \mathrm{~nm}$ are consistent with tolerances on readily available optical components. To reduce the degree of non-linearity below that required by the EUVOMM, however, requires the introduction of new lechniques which are described later.

The surface sensing mechanism of the proposed instrument is basod on the constant force profiler developed at the University of North Carolina.[?] This instrument bridges the performance gap between conventional stylus profiling instruments and the short range atomic force microscopes presently available. The sensing head has already been demonstrated in a configuration which provides a $15 \mu \mathrm{m}$ vertical range and a $50 \mathrm{~mm}$ profile length. The probe tip consists of a thin glass cantilever on which is mounted a diamond tip. The back surface of the cantilever provides one plate of a capacitance gauge. The other plate of the capacitance gauge is supplied by the Zerodur ${ }^{\mathrm{TM}}$ support block on which the cantilever is mountcd. The use of Zerodur ${ }^{\mathrm{MM}}$ for the structure of the tip perrnits the rear surface of the probe assembly to be used as a reliable reference.

\section{Probe Head Assembly}

Stylus and constant force transducers form the basis of a broad range of instruments for metrological characterization of fine surface features.[9.10] Typically, stylus based instruments consist of a long range traverse mechanism for translation of the specimen relative to the probe, with the probe system mounted with its axis of measurement perpendicular to the traverse direction. In practice, it is the Abbe errors associaled with the misalignment between the axis of the probe that interrogates the surface and the measurement axis of the transducer used to sense the displacement of the probe that dominate the achievable precision in profile beight measurement. For this instrument, a force probe system is being developed to measure the optical surfaces with an 
accuracy of $1 \mathrm{~nm}$ and a resolution of $0.1 \mathrm{~nm}$ for vertical surface profile deviations of up to $3 \mathrm{~mm}$. The heart of this profiler consists of a constant force probe similar to that developed by Howard and Smith, $\left[{ }^{[1,12]}\right.$ combined with a laser interferometer as described above. Since the Abbe error is due to both the offset and the angle of tilt, control of either parameter can be used to minimize this error. The precision of alignment between the axis of the laser interferometer and the stylus probe is limited by manufacturing considerations. The stralegy used to minimize this error is to control the angle of tilt. To this end a novel, three-axis flexure arrangement has been devised that allows for active control of two rotations over a range of $30 \mu \mathrm{rad}$ while allowing a displacement of approximately $1.5 \mu \mathrm{m}$. . The tilt control mechanism may also be exploited to obtain an estimate of the Abbe offset by monitoring the displacements as measured by the laser and the probe while rocking the probe head through a known angle.

A schematic diagram indicating the relationship between the various parts of the device is shown in Figure 3. The force probe is mounted to the target mirror assembly which, in turn serves as a plane nirror for a remote interferometer. The mirror assembly also functions as a target for capacitance gages. The PZT actuators can be controlled individually in combination to provide tilt and translation of the force probe relative to the top plate. Since tilt in two axes is required, a minimum of three actuators and three capacitance gages are required.

The force probe assembly used to interrogate the surface is shown in Figure 4. It consists of a stylus probe mounted on a glass cantilever. Electrodes deposited on the back of the cantilever and the top of the base form the plates of a capacitor. Deflections of the cantilever change the spacing between these plates, changing the capacitance, thus enabling the deflections of the cantilever to be monitored. This design is bred on the work of Howand and Smith and is capable of sub-nanometer resolution over a $1 \mu \mathrm{m}$ range.

In addition to providing tilt cancellation, the probe head also serves as a high-bandwidth actuator which is used to provide constant force stylus measurement. This is achieved by servo controlling the extension of the probe head to maintain constant cantilever deflection. The probe head in turn is mounted to a low-bandwidth, long-range vertical slide which provides the travel required by the large vertical profile deviations envisaged in current applications. For absolute measure ments, the position and tilt of the force probe is measured relative to a metrology frame by interferometric methods. For this purpose, the rear surface of the probe is an optically nat reflector.

The force probe assembly is mounted to the probe head which consists of the actuators, flexures and capacitance displacement sensors. The flexure consists of two sets of three flexure elements (represented schematically in Figure 5 by the springs). The lower set of fexures provide the compliance for the PZT actuators to enable the required displacement and tilt. The upper set is used to adjust the preload on the actuators during assembly and allow for slight dimensional mismatch between the length of the flexure and that of the actuntors. The adjustment screws control the deflections of the more compliant upper flexures which in turn control the preload on the actuators due to the deflection of the lower flexures. Once the preload is set, the controlling stifrness is that of the lower flexures. The deflections of the individual PZT actuators are monitored by capacitance gages which can be used in combination to measure both displacement 
and tilt of the probe. Although the position of the force probe is monitored by a remote interferometer, the capacitance gages provide a more tightly coupled metrology loop that enhances the controllability of the entire system. In addition, the present design allows for the operation of the device as a stand alone device and facilitates bench lesting.

Figure 6 shows an exploded view of the complete assembly. The two sets of flexures are part of a monolithic structure made out of aluminum which also includes slots for the PZT Bctuatore. The flexures are equispaced around the circumference of a circle giving the monolithic flexure three-fold symmetry. Three PZI actuator segments are mounted on the top plate and pass through slots in the flexure. The actuators are made from a single piece of ceramic and exploit the Poisson effect to produce the desired fine motion. These actuators bear on the boltom plate via spherical contact surfaces that allow for the angular tilt. Capacitance gages mounted in the top platc monitor the displacement of each PZT' with the bottom plate forming the target for the capacitance gages. The force probe is mounted to the bottom of the target mirror, which in turn is clamped to the lower surface of the bottom cap using a semi-kinematic clamping arrangement (not shown). Changes in length of the PZT actuators cause the bottom plate to tilt and translate relative to the top plate, the latter of which has a large aperture to allow the laser beams of a remote interferometer to pass through to the target mirror. While the aluminum structure of the probe head is susceptible to thermal changes, the force probe assembly and target mirror are constructed out of Zerodur ${ }^{\mathrm{m}}$ and are directly referenced to the metrology frame through the interferometer, thus minimizing the thermal sensitivity of the main measurement loop. Monolithic construction has been employed to minimize joint losses and hysteresis and to obtain a compact, high stiffness structure.

\section{Metrology Subsystem}

To achieve the resolution and accuracy of the EUVOMM requires very tightly controlled feedback loops to relate the geometrical configuration of the machine to a highly accurate standard. Careful attention must be paid to those portions of the mechanical system outside of the loops, as well as the relationship between loop-controlled dimensions and the output of the metrology subsystem. All of these concerns in turn depend upon the metrology subsystem possessing a reference of appropriate accuracy, resolution and dynamic range. The most realistic candidate for this task is Distance-Measuring Interferometry (DMI). Several companies sell DMI's utilizing Michelson's interferometer configuration which is schematically shown in Figure 7 and termed "Homodyne DMI" to emphasize the analogy with radio technology.

A single frequency light beam is split into two paths and then recombined. The detector sees one cycle of intensity variation each time the difference in the two optical paths changes by one wavelength. By counting these cycles, changes in the optical path difference is thereby determined in wavelength units. This architecture suffers from the following limitations: 1) When the optical path is changing slowly the intensity variations take place near $0 \mathrm{~Hz}$ where there are many l/f noise mechanisms active, such as variations in the optical alignment or light source intensity, which can not be distinguished from variations due to the desired optical path changes 2) These noise sources make interpolation of fractional wavelength changes very difficult, thus 
limiting resolution 3) When the intensity is near a peak or valley there is no simple way to determine in which direction the optical path is changing. A method to overcome these problems was provided commercially by Hewlett Packard. This solution, indicated in Figure 8, is termed a "Hcterodyne DMI" and utilizes two different optical frequencies; one for each of the two optical paths. This causes variations in optical path difference to appear as phase variations between the beat note of the two optical frequencies before they enter the beam splitter and the beat note after they are recombined. This beat note is placed at a portion of the spectrum ( $2 \mathrm{Mhz}$ for HP) which is much quieter than $0 \mathrm{~Hz}$. Dircction then appears as an advancing or retarding phase shif.

In spite of the great improvement in resolution and robustness that heterodyne DMI's provided, major potential applications that desired the performance were deterred by the cost and by the difficulty in achieving and maintaining optical alignment. The first obstacles encountered when allempting to achieve greater performance in position resolution are "optical non linearity or periodic error" and "electronic non-lincarity'. The former is caused by imperfect separation of the two optical frequencies wherein some of $f 1$ traverses the path intended only for $f 2$ and vice versa. The latter is caused by parasitic couplings between phase locked loops in proximity to one another (injection locking), or by the difficulties in obtaining precise nanosecond time delays . Starting around 1980 many papers began to appear analyzing and measuring periodlc error. In 1988 one of the authors developed a very general and simplified analysis of polarization mixing which made evident an electronic means for canceling most of its effect without eliminating the underlying cause. This technique was implemented on five of the seven axcs of LODTM at LLNL. Although it provides a major reduction in periodic error it has to be "hand tweaked" from time to time for each axis of motion. This requirement along with the increased accuracy demands of EUVOMM led to the search for another approach. We concluded that it might be practical to avoid the fundamental cause of the problem altogether by utilizing spatial separation of the two opucal frequencies instead of trying to maintain their polarization orthogonality. Figure 9 illustrates this concept:

Optical frequency $\mathfrak{n}$ enters the interferometer and is split into two beams. Then each of these is combined with a portion of $f 2$ upon leaving the interferometer on the way to the two detectors. The phase shift between the beat notes from the detectors provides the desired information. There is no need to maintain polarization control to keep $f 2$ out of the oplical paths intended for $f$ only. Another way of looking at this is that 2 serves as the local oscillator frequency to translate the frequency of $\mathrm{fl}$ to that of the difference in their optical frequencies, just like a heterodyne radio. The following summarizes the features: 1) No periodic error 2) No need to maintain tight polarization control 3) More than one frequency can follow the path of $f$ and then be separated, afler translation by $\mathfrak{Z}$ to the electronic range, with frequency-domain filters. These signals can be combined to form the basis of an Absolute Interferometer over limited ranges or they can be used to self-check the linearity of the electronics. It is possible that they might be used to enable the use of an untuned laser by averaging the wavelengths of the many longitudinal modes present.

The relaxing of polarization control has several consequences: a) it eliminates polarization considerations during optical alignment b) it reduces the cost of the optical components c) it makes a fiber-optic light distribution system much more feasible because the polarization degradation caused by the fibers is no longer important. A fiber feed greatly simplifies the usually 
very difficult task of optical alignment since each axis is now independent of the others (this is particularly applicable for LODTM). The only alignnent necessary is parallelism of the optical beam to the axis of motion (cosine error).

To take advantage of the additional information provided by this optical hardware, electronics are required that can measure millions of cycles of phase shift and then resolve the final cycle into as many parts as possible. The phase accuracy of the existing commercial circuitry is limited to around $1 / 256$ of a cycle due to injection locking of the phase locked loops. In order to meet the requirements of EUVOMM a new electronic architecture was developed, see Figure 10. utilizing components recently developed for the massive cellular market.

Computer simulation of this architecture has been performed assuming various noise sources and an ideal $A$ to $D$ converter The results of simulation indicate that electronic resolution of order several thousand may be possible. This resolution meets EUVOMM requirements. 
I See, for example, M. Born and E. Wolf, Priniciples of Oplics, 9.1.3, Pergamon Press, NY, 1964.

2 D. Steams, "Issues in multilayer technology for SXPL," Proceedings of the Flrst Technical Symposium on Soft $X$-ray Projection Lithography, Asilomar Conference Center, Poxific Grove, CA Jan 1990.

3 D. WInd, "XUV Multilayer Program at AT\&T," Proceedings of the First Techrical Symposium on Soff X-ray Projection Lithography, Asilomar Conference Center, Pacine Grove, CA Jan 1990.

- U.S. Patent No. 5,019,786, "Phase Measurement System Using a Dithcred Clock"

- A. E. Rosenbluth and N. Bobroff, "Oplical sources of non-linearity in heterodync interferometers," Precison Engineering, 12,17-11, Jan 1990.

- N. Bobroff, "Recent advances in displaccment measurlog interferometry," Measurment Sclence and Technology, $4907-926$ (1993).

1 L. P. Howatd, S. T. Smith, "Long range constant force profiling for measurement of enginecring surfaces," Review of Scientifle instrumenus 63(10), 4289-4295 (Oct 1992).

- Thomas, T. R., Rough Surfaces, Longman Press, London, 1982.

9 Chetwynd, D. G., and Smith, S. T., "High precision surface profilometry: From stylus to STM,", Fram instruments in Nanotechnology, ed. Gardner, J. W., and Hingle, H. T., Gordon and Breach, London, 1991, pp. 273-300.

10 Sarid, D., Scanning furce microscooy: with applications to electric, mesnetic and alomic forces, Oxford University Press, NY, 1994.

"Howard, L. P., and Smith, S. 1., "A mctrological constant force profiler," Rev. Sci. Instrum., 65(4), 1994, pp. 892-902.

12 Smith, S. I', and Howard, L. P., "A precision, low force balance and its application to atomic force probe characterization," Rev. Sci. Instrum., 65(4), 1994, pp. 903-903 


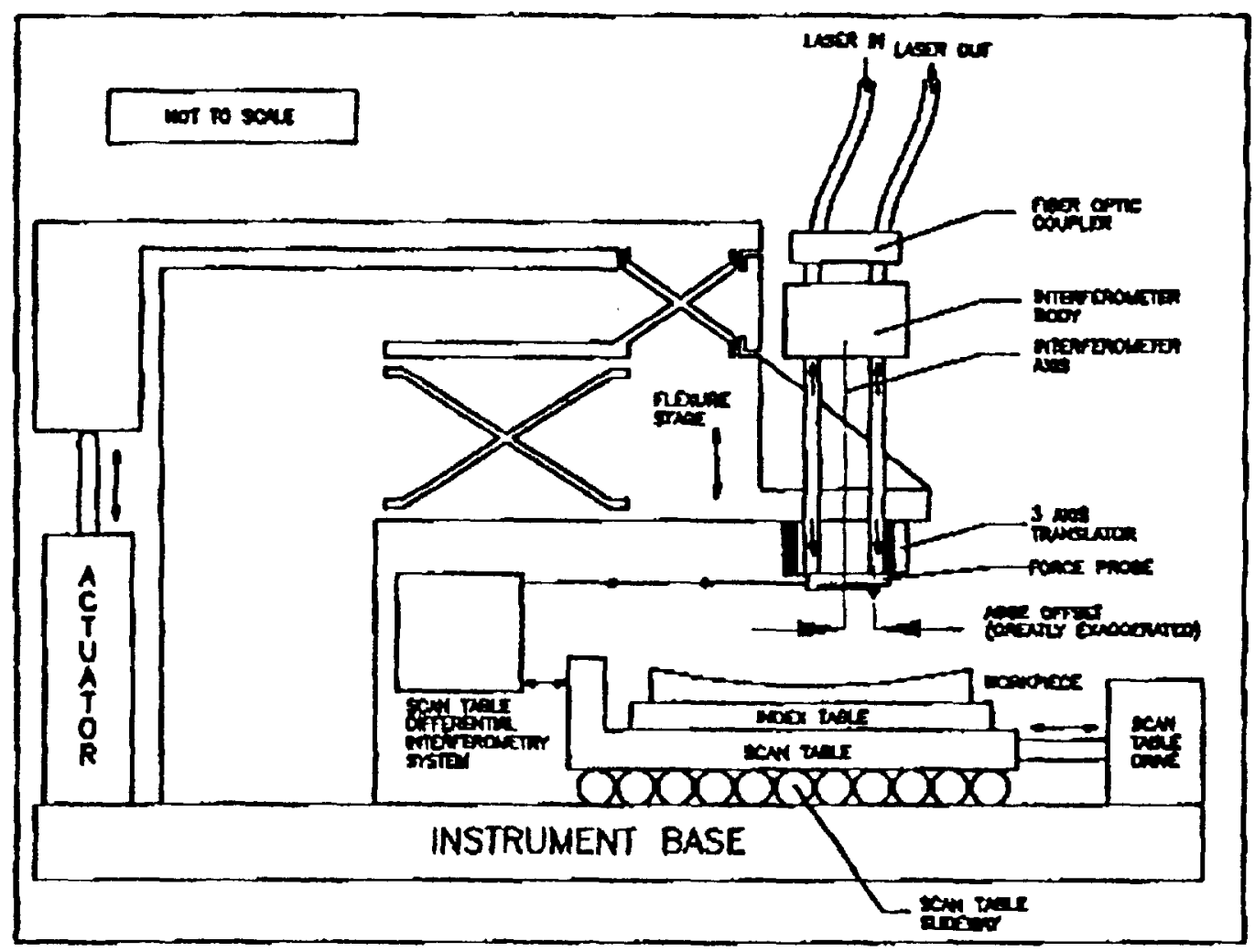

Figure 1 Eurowy design concept. 


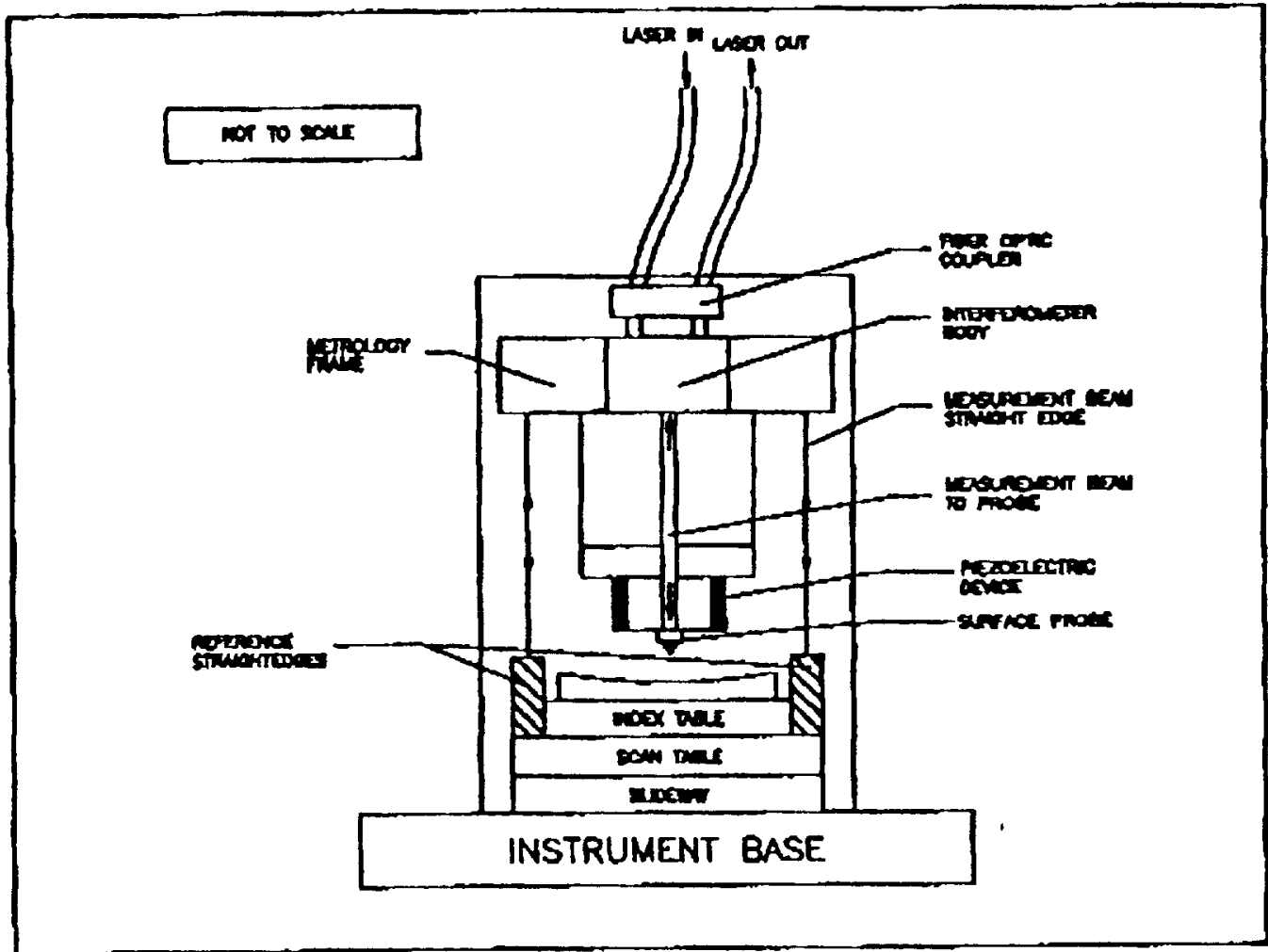

Figure 2 Exram metbology system for vertical measurements. 


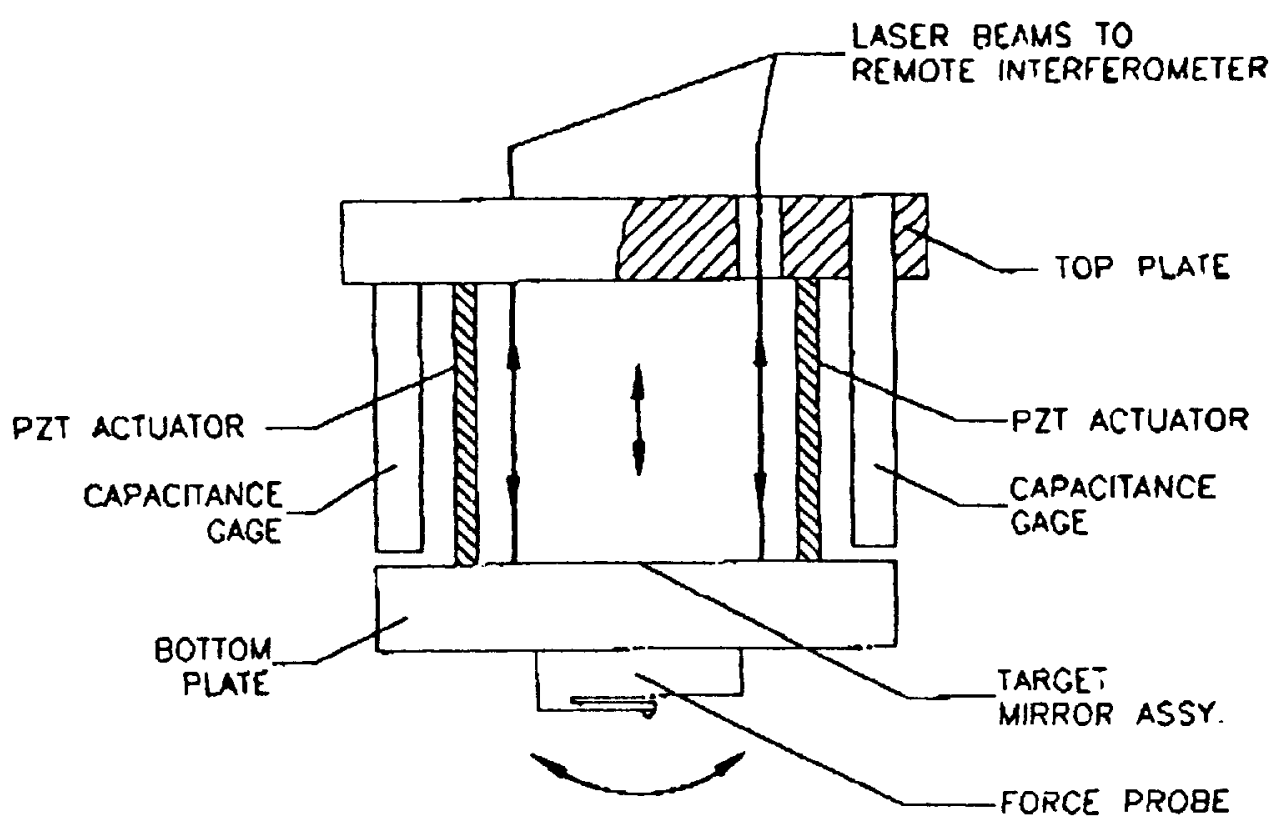

Figure 3 Relative dispositions of capacitance gages, interferoneter and PZT's in probe head. 


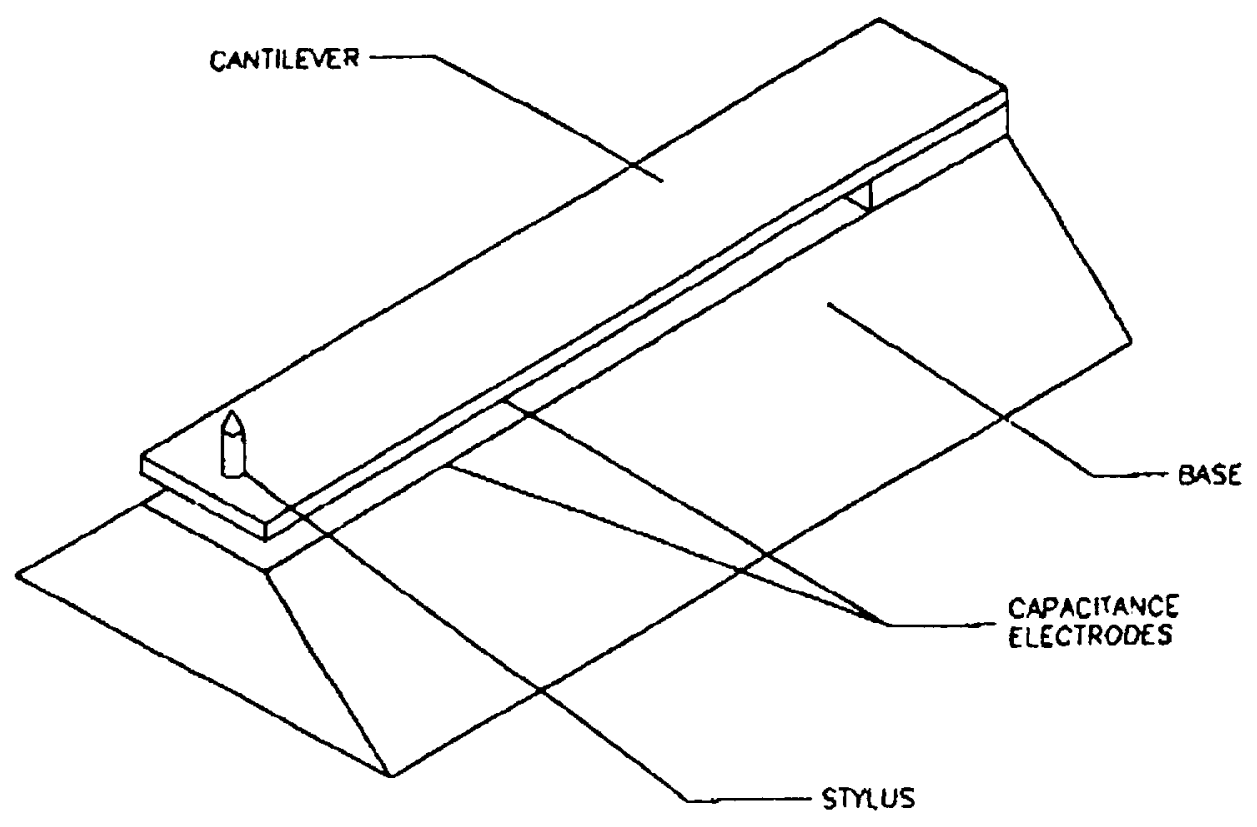

Figure 4 schematic of force probe. 


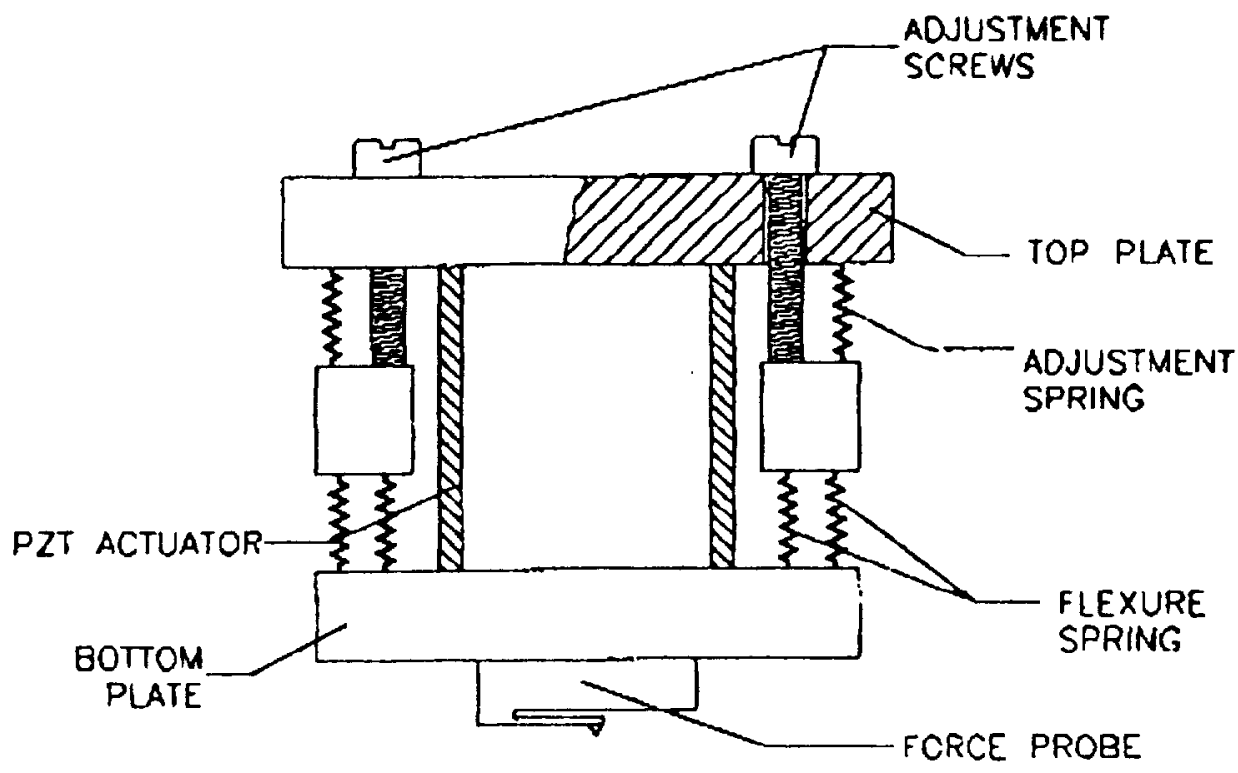

Flgure 5 schematic of flexure arrangement of probe head. 


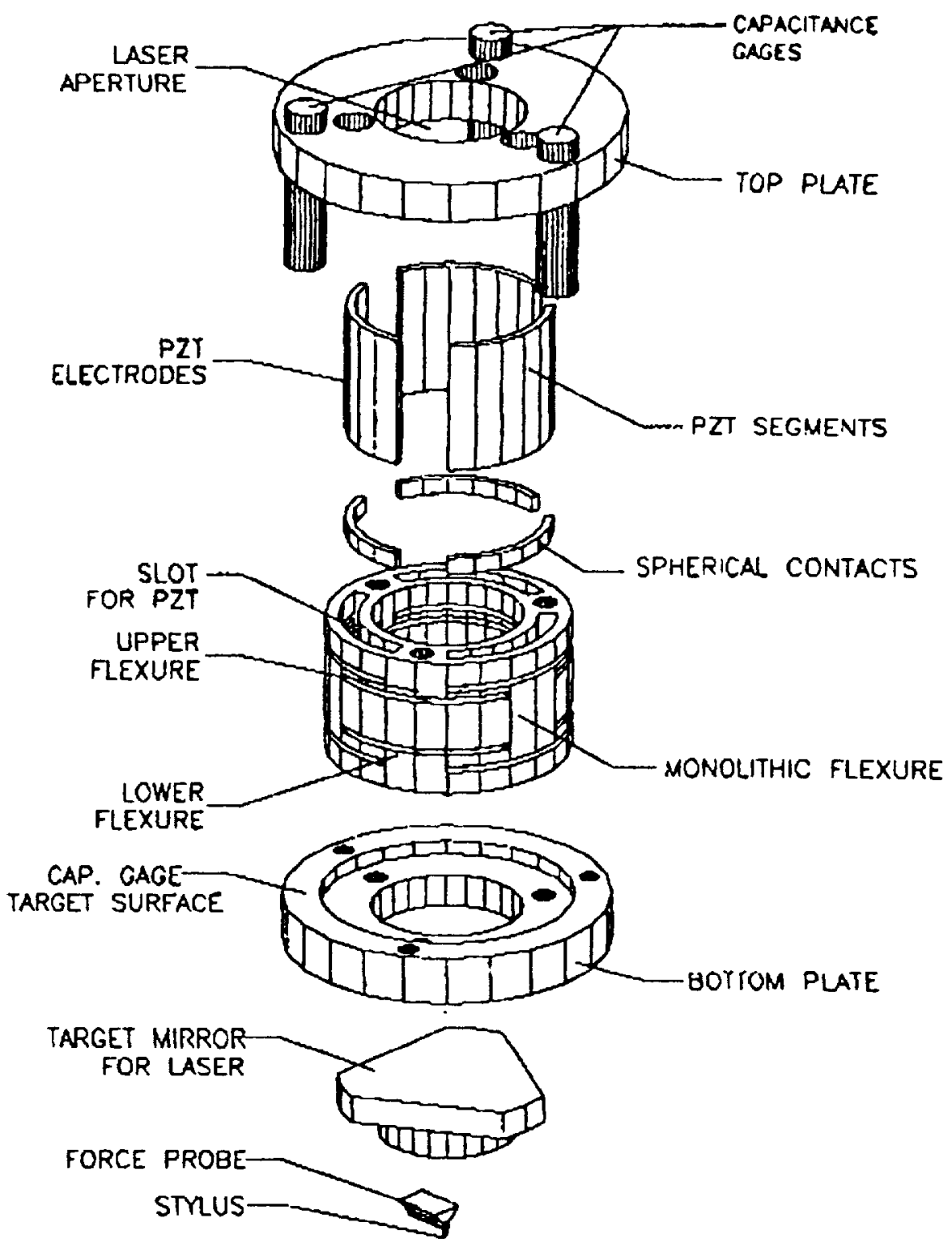

Eigure 6 Exploded view of Probe Head Aasembly. 


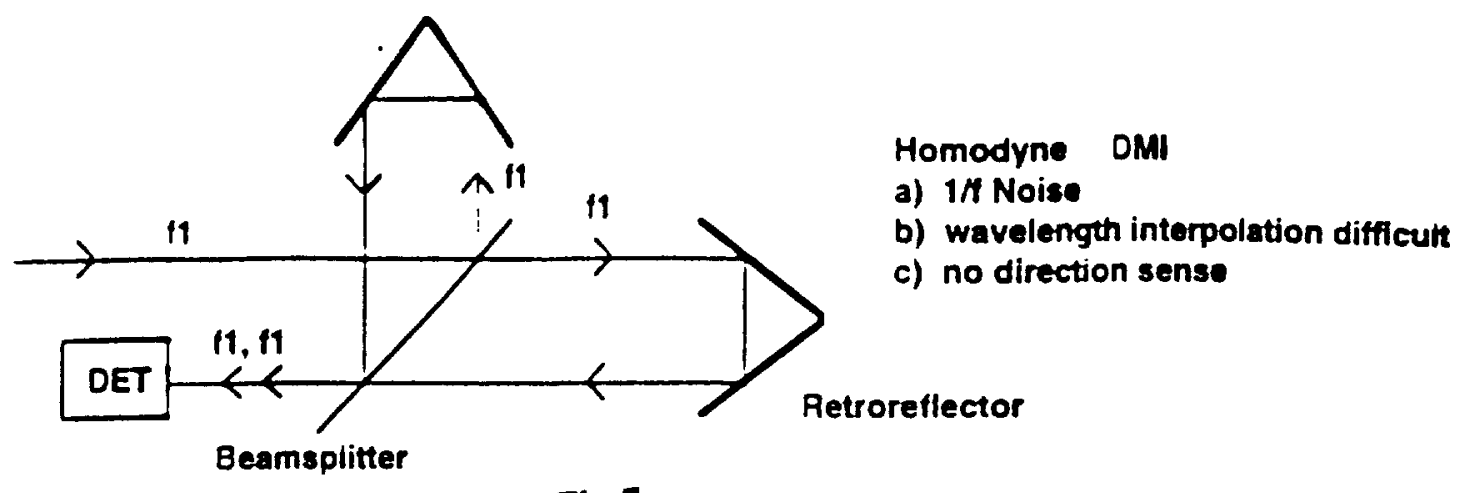

Fig 7 


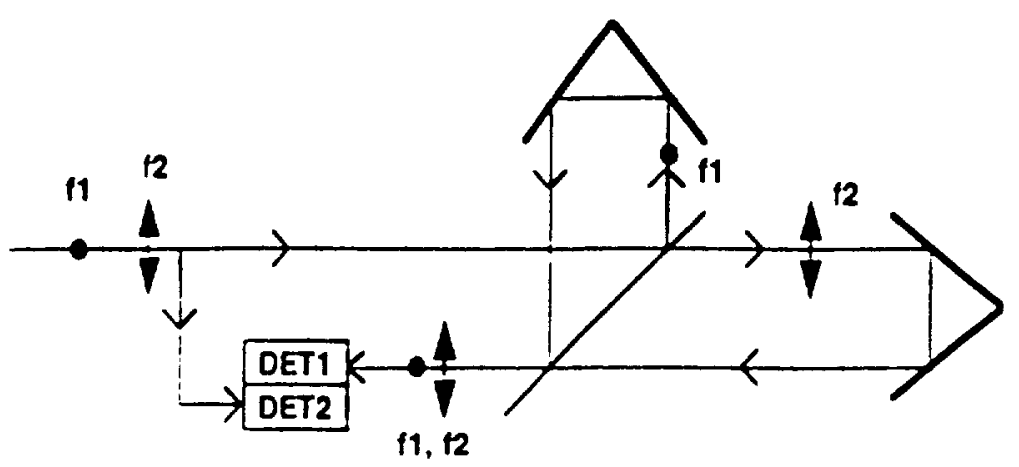

Fig 8
Polarization-onthogonal

Heterodyne DMI

a) imperfect separation of 11,12 causes errors 


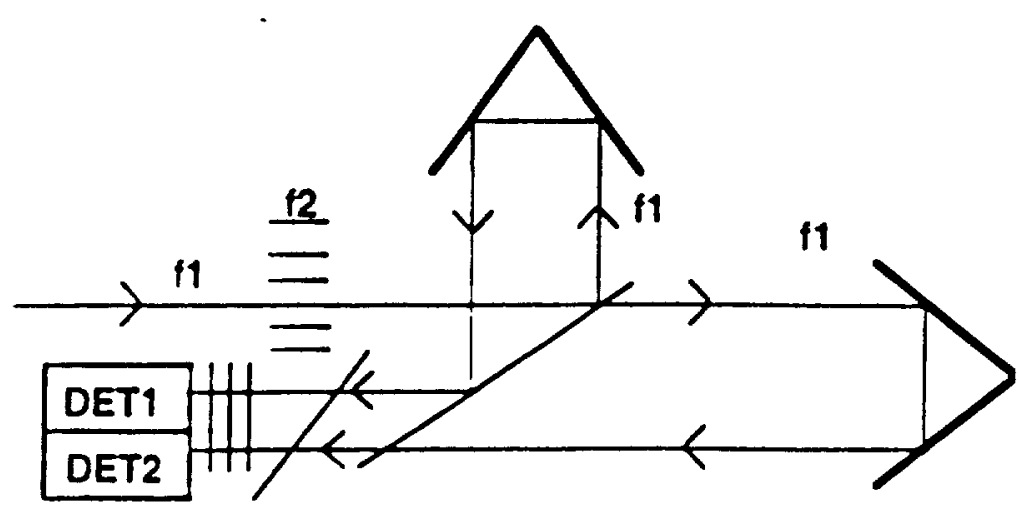

Fig 9
Spatially-Orthogonal

Heterodyne DMI 
A very promising electronic architecture, based upon chips developed for cellular communications, has been conceived

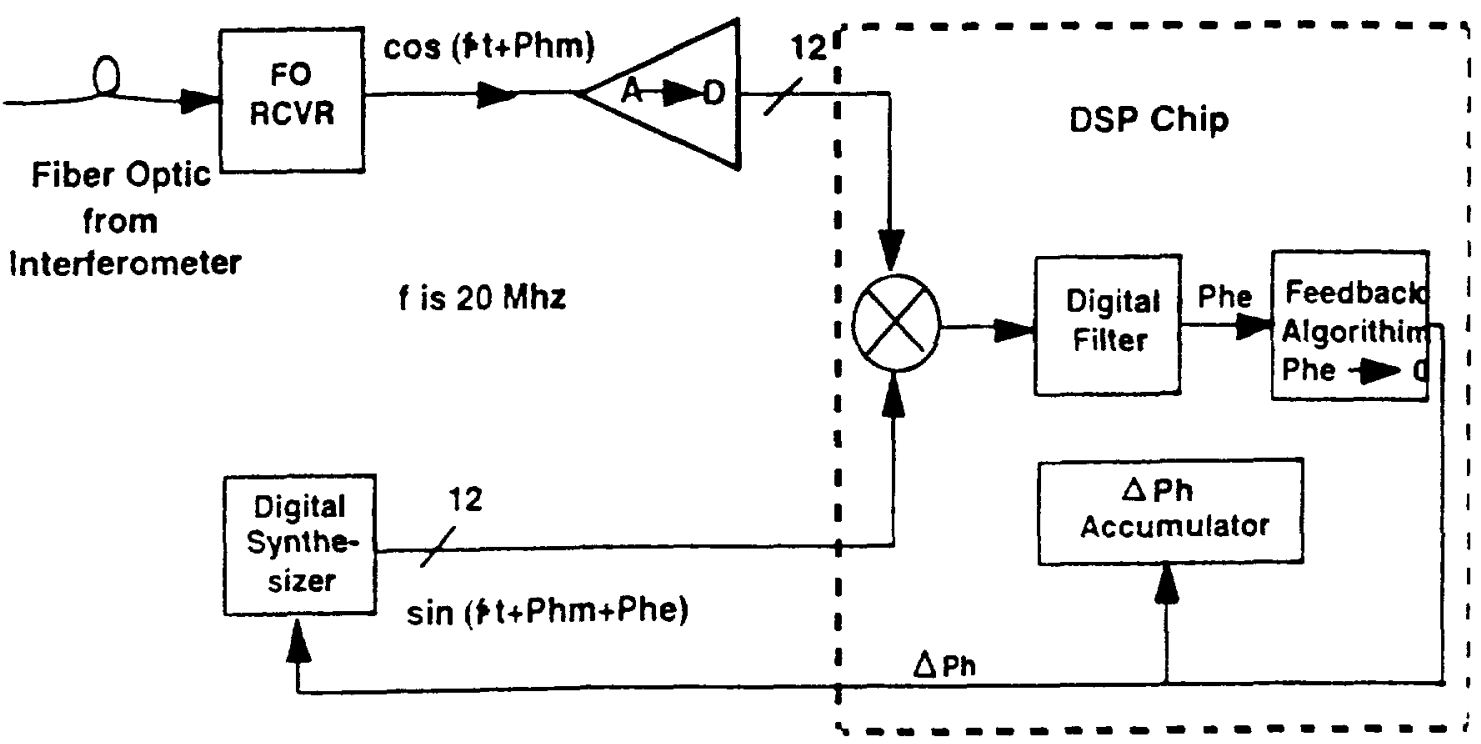

Fig 10 


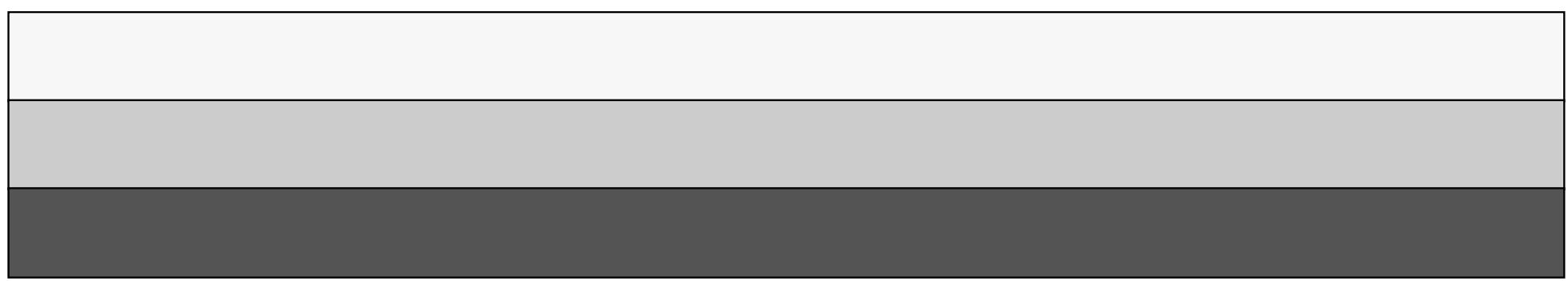

